\title{
A Markov model for estimating the remaining life of electrical insulation in distribution transformer
}

\author{
${ }^{1}$ A.U. Adoghe, ${ }^{2}$ C.O.A. Awosope and ${ }^{1}$ J.C. Ekeh \\ ${ }^{1}$ Department of Electrical \& Information Engineering, College of Science and Technology, \\ Covenant University, Ota, Nigeria, adoghe tony@ieee.org, Mobile phone-234-803-3885-086. \\ ${ }^{2}$ Department of Elect/Elect, Faculty of Engineering, University of Lagos, Lagos, Nigeria
}

\begin{abstract}
In this paper, a Markov maintenance model for predicting the remaining life of transformer insulation has been developed. It incorporates various stages of insulation deterioration and minor maintenance state. Given that a current state of insulation ageing has been reached, from a diagnostic testing and inspection, this model is capable of computing the predicted average time before insulation failure occurs. The algorithm developed in this model was implemented in a computer program using Matlab. The data required for this program and the numerical example based on the developed algorithm are also discussed
\end{abstract}

Keywords: maintenance model, transformer insulation, distribution transformer, markov model, developed algorithm.

\section{INTRODUCTION}

Prediction of the remaining life of distribution transformers is an important issue for utilities companies because of the need for planning maintenance and capital expenditures. Transformer is the most expensive equipment in electrical network. The un-scheduled outages of the transformers due to unexpected failures are catastrophic in many cases.

Transformer management activities are many and researchers tackle them differently. Maintenance planning and condition monitoring techniques are examples of the general management activities that can be applied to any equipment such as transformer, oil circuit breaker, protective equipment, etc. Asset management activities is however different from equipment to equipment. Condition monitoring techniques applied to transformers for example are different from those applied to oil circuit breakers or protective equipment although some of these techniques may have similarities. Major losses involving large oil-cooled transformers continue to occur on a frequent basis. The reasons being that there are increased equipment utilization, deferred capital expenditures and reduced maintenance budgets.

Asset management systems have been developed to assist asset managers in optimizing the service availability of these important network assets within the available budget by making informed maintenance decisions [1]. The quality of these decisions depends significantly on the accuracy and efficiencyof the deterioration models used to predict the remaining life of network components [2]. A deterioration model is defined as a link between measures of facility condition that assess the extent and severity of material damages, and vectors of explanatory variables that represent the factors affecting facility deterioration, such as age, material properties, applied loads, environmental conditions, etc [3]. Several deterministic and stochastic approaches have been developed to model component deterioration [4].

In this paper, a probabilistic model for predicting the remaining life of electrical insulation deterioration in distribution transformer has been implemented. The development of the Markov model is presented in section 2 and this is followed by the development of the algorithm for the computer program in section 3. The discussion and solution of the numerical solution based on the computer program followed by the conclusion are presented in section 4 and 5 respectively.

Developing the Markov Model for estimation of the remaining life: For this system under study, four states can be identified with reasonable accuracy:

(a) Normal or working state

(b) Minor deteriorating state 
(c) Major deteriorating state and

(d) Failure state

In developing this model, we will assume that the system, if not maintained, will deteriorate in stages (for a general model, $\mathrm{k}$-deterioration stages are assumed) and will eventually fail at $k+1[5]$. Failure can also occur as a result of other causes not associated with typical ageing and we will call such a failure a random or poisson outage. If the deterioration process is discovered, preventive maintenance is performed which is expected to restore the system back to the original condition of deterioration (assumed).
Repair maintenance after either random or deterioration induced failure will restore the system to as new condition.

All these assumptions are incorporated in the developed state -space Markov model. A model based on discrete parameter (succession of events) is presented.

This system is described through the transition probabilities $F_{6} /$ indicating the probability of moving from state i to state $\mathrm{j}$ in a given time interval, $\Delta^{T}$.

Markov model representing various stages of deterioration that will eventually culminate in failure is presented [6]. This model is presented in figure 1.

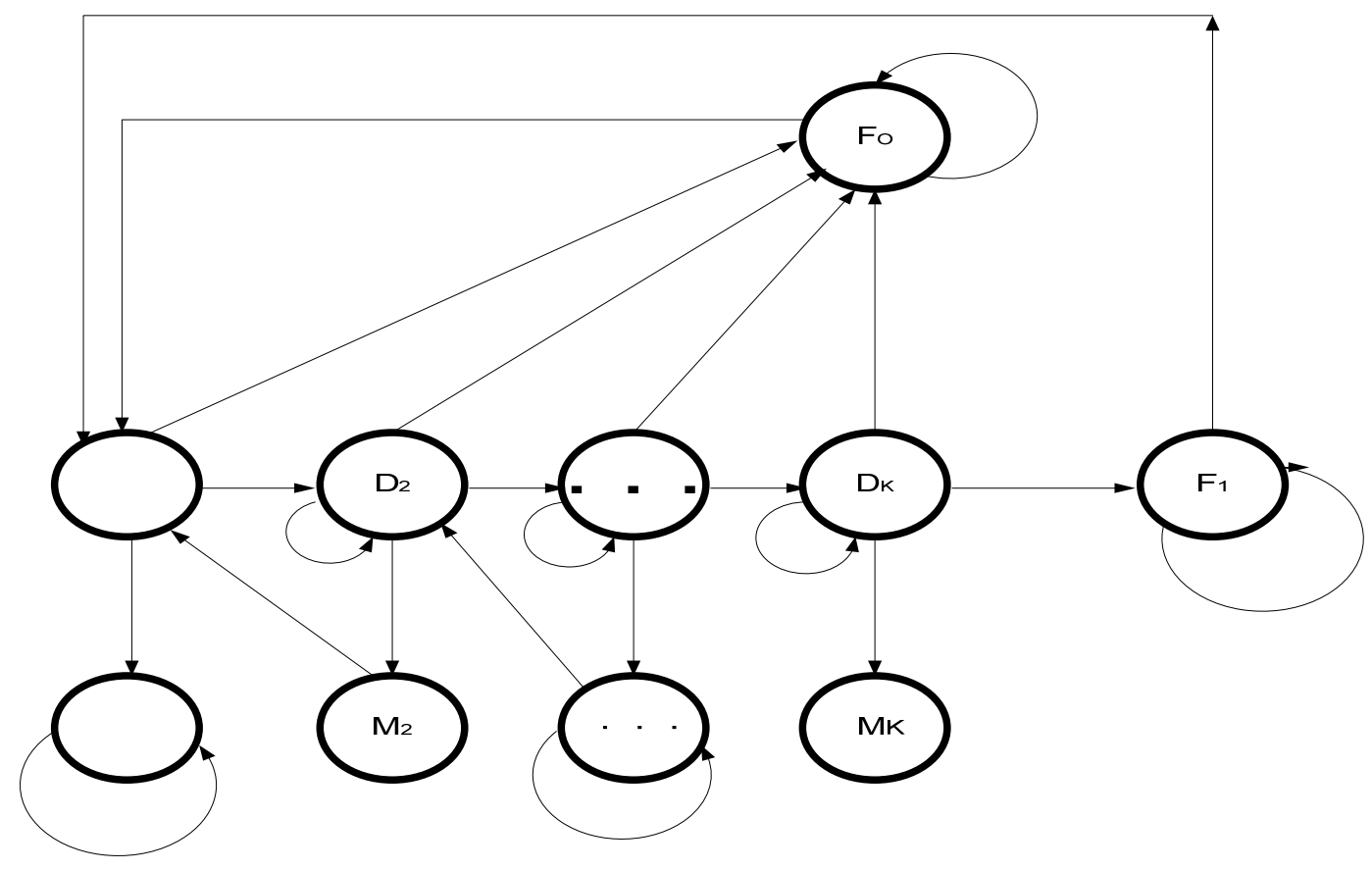

Figure 1 Discrete - parameter Markov model for determination of the remaining life

In the model shows in figure $1, D_{1} \ldots D_{k}$ denote deterioration states, with $D_{1}$ being the normal state $M_{1} \ldots M_{k}$ denote maintenance states respectively. The computation of the expected transition time from any of the system states to state $F_{1}$ (expected remaining life) can be performed using standard Markov techniques as outlined below:
Transition probability matrix $P=\left[P_{4 i j}\right]$ is constructed from inspection /observation data of the identified critical component. Here i and j represent indices of all the states.

The constructed matrix $\mathrm{P}$ can be partitioned into four sub-matrices $F=\left[\begin{array}{cc}Q & R \\ O & T\end{array}\right] \quad$ where $\pi=F\left(F_{1} F_{1}\right)$ since state $F_{1}$ is the last in the state array. 
From here, Matrix $\mathrm{N}$ called the fundamental matrix of the Markov chain is constructed from $P$.

i.e $\quad A=(I-\varphi)^{-1} \quad$ where $\mid$ represent identity matrix and $\mathrm{N}$ is called the fundamental matrix of the Markov chain. $W_{t j}$ represents the $t^{t h}$ element of $\mathrm{N}, T_{i}$ the sum of the entries in row $\mathrm{i}$ of $\mathrm{N} . b_{i j}$ the $i f^{\text {th }}$ entry of the matrix $B=N R$.

- The number $w_{t}$ is the average number of times the process is in the $i^{\text {th }}$ transient state if it starts in the $i^{\text {in }}$ transient state.

- The number $T_{i}$ is the average number of steps before the process enters an absorbing state if it starts in the $i^{\text {th }}$ transient state.

- The number $B_{i j}$ is the probability of eventually entering the $i^{\text {th }}$ absorbing state if the process starts in the $t^{\text {th }}$ transient state.
It can be shown that the elements of $\mathrm{N}, N_{t} /$ give the mean number of visits starting from state $i$ to a transient state $\mathrm{j}$ (deterioration or maintenance state) before entering a deterioration failure state [7].

Therefore, if $M_{i}$ is the expected remaining life of the component if the system is in state $i$, it can be expressed

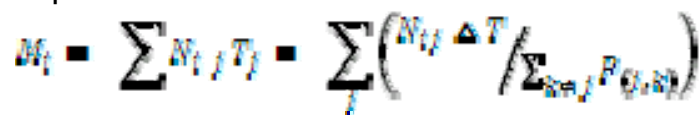

where $T_{j}$ is the mean time spent in state $\mathrm{j}$.

Methods based on continuous time: In engineering practice, it is often easier to determine transition rates than transition probabilities. A Markov model for the evaluation of the remaining life of insulation based on continuous parameter is shown in figure 2 .

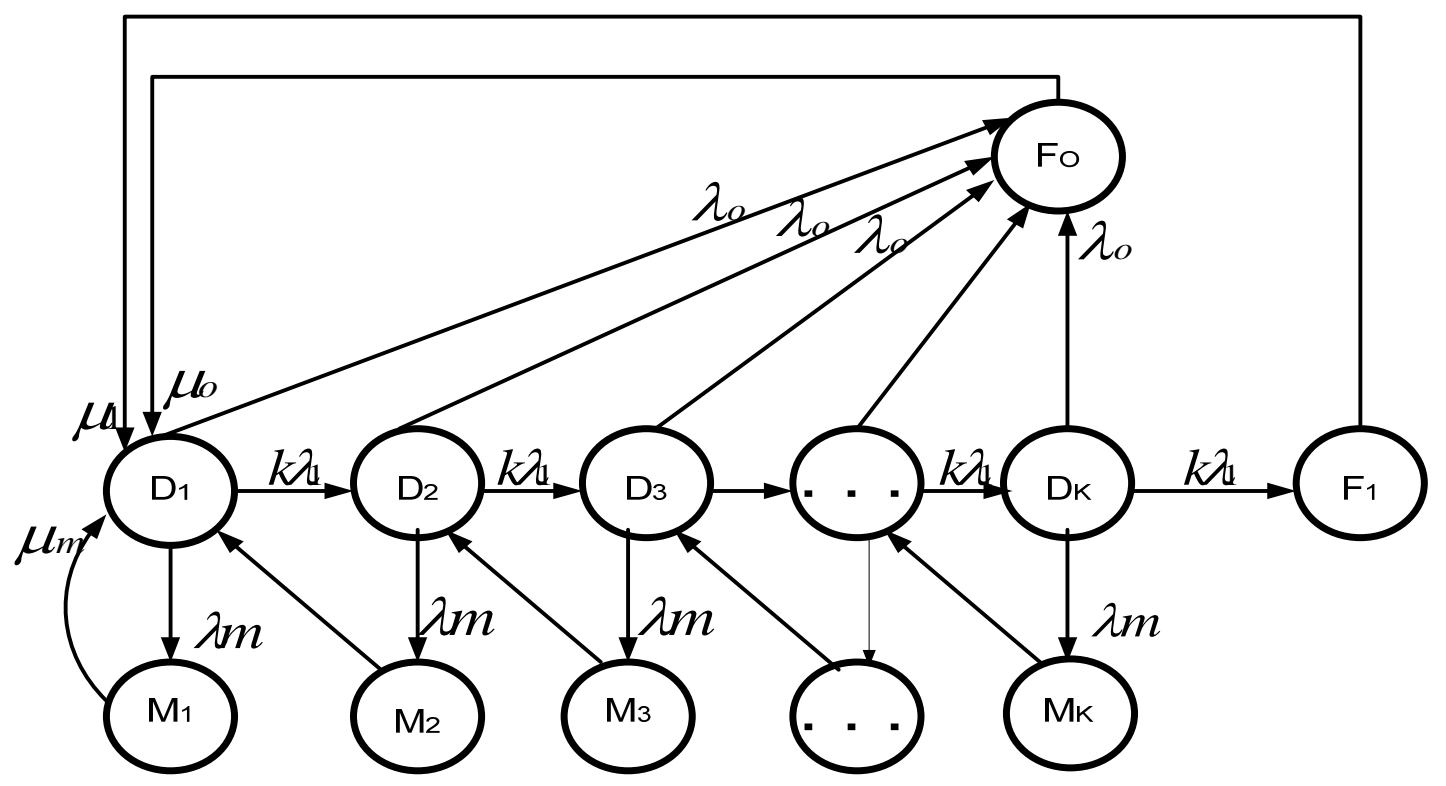

Figure 2 Continuous parameter Markov model

Determination of the transition rate parameter: All parameters can be estimated from historical records, except for $\lambda$, the reciprocal of the mean time to failure if no maintenance is carried out.

To obtain the value of $\lambda$, proceed as follows:

- Observe the average time to deterioration failure $T_{f}^{*}$. This is the average time between failure events and it can be easily recorded.
- Solve the Markov model for various values of $\lambda$, to obtain the function shown in figure 3 .

- From this function, determine the values of $x^{*}$ corresponding to the value of $T_{f}^{*}$ recorded earlier. 
Am. J. Sci. Ind. Res., 2010, 1(3): 539-548

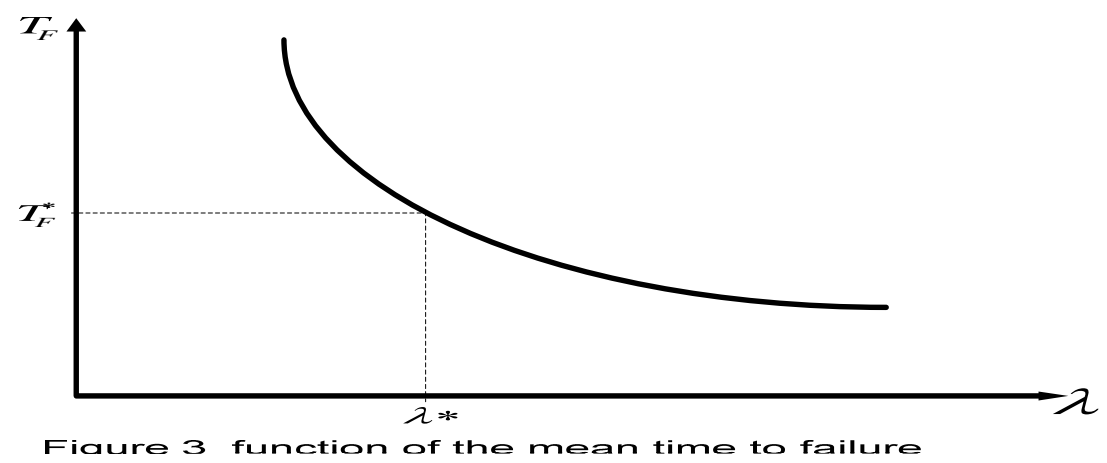

To determine the expected time for a component failure, figure 2 can now be reduced to figure 4

where transition rates are used instead of transition probabilities.

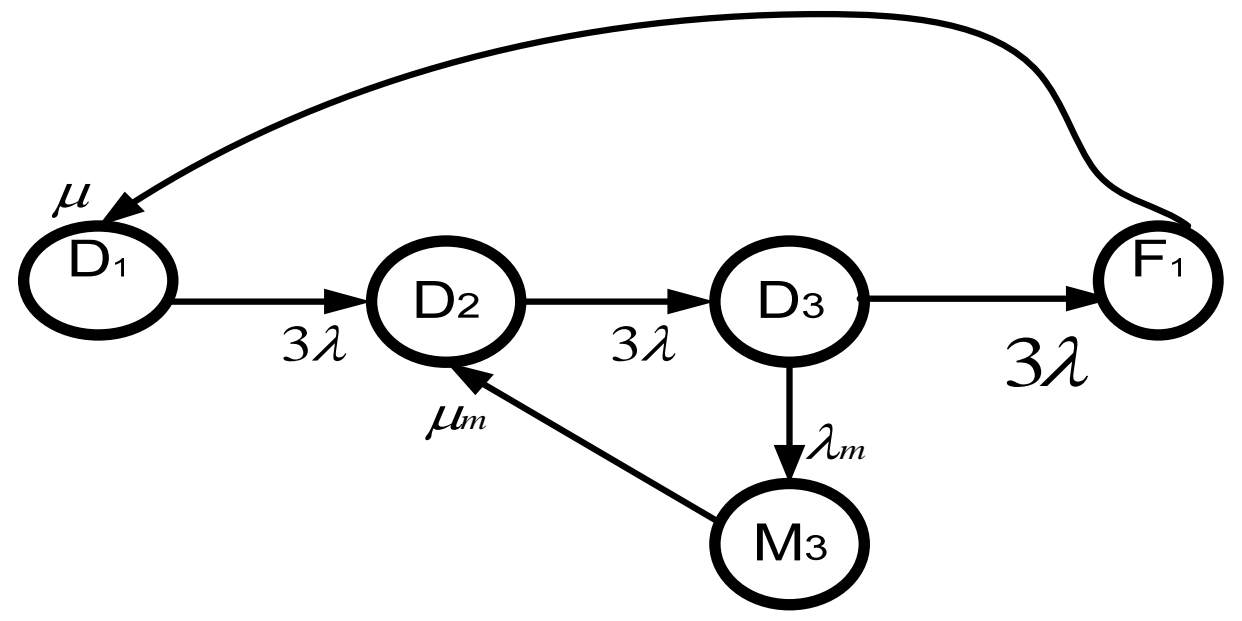

Figure 4 Markov model with continuous parameter.

For $\mathrm{k}=3$, we also denote state $D_{2}$ by i and state

$F_{1}$ by j. Applying the rules for state combination

[8] as illustrated in figure 5 , we have

$$
\begin{aligned}
& \lambda_{i s}=3 \lambda, \quad \lambda_{i s}=\mu \\
& \lambda_{l 2}=\frac{P_{D 1} 3 \lambda+P_{M 8} F_{M}}{P_{D 1}+P_{D 8}+P_{M \gamma}} \\
& \lambda_{g j}=\frac{P_{D \delta} 3 \lambda}{P_{D 1}+P_{D 8}+P_{M z}}
\end{aligned}
$$$$
\text { ..... } 1
$$

where the probabtittes $P_{D 1}, P_{D S}$ and $P_{M 3}$ are the steady - state probabilities of the system states indicated. 
Am. J. Sci. Ind. Res., 2010, 1(3): 539-548

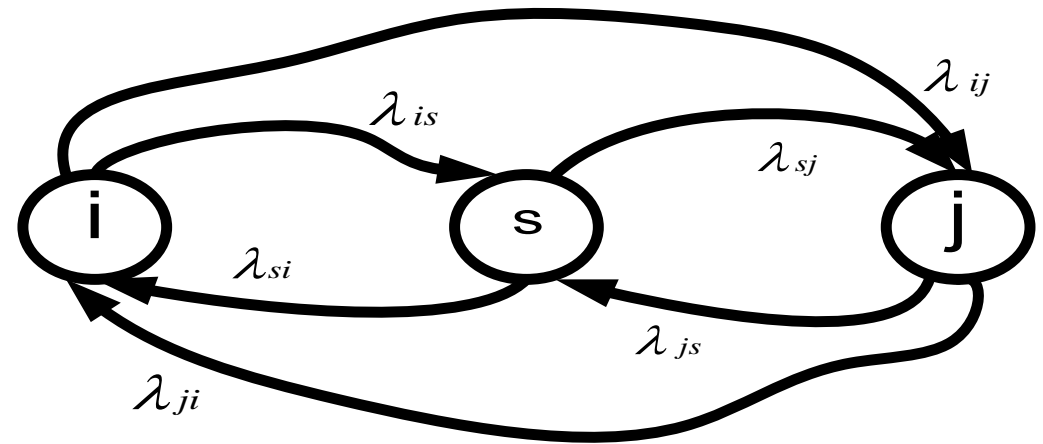

Figure 5 Diagram illustrating development of the mean transition time between states $\mathrm{i}$ and $\mathrm{j}$.

Determination of steady states probabilities: The steady - state probabilities needed in equation (1) are obtained by solving the equations $P . Q=O$ The $p_{i}$ are unknown, they are the values we wish to find since they are the steady- state probabilities of the system states indicated in figure 2. If there are $\mathrm{n}-$ states in the state space, there are $n$ such equations in $n-$ unknowns. Unfortunately, this collection of equation is irreducible. We need another equation in order to solve the equations and find the unknowns. Fortunately, since $\left\{p_{i}\right\}$ is a probability distribution, we also know that the normalisation condition holds.

$\sum_{n_{i}=5} F_{i}=1$

These $n+1$ equations, can be solved to find the $n$ unknowns $\left\{\mathrm{P}_{\mathrm{i}}\right\}$

Where $\mathrm{Q}$, the transition intensity matrix, is generated from the state transition diagram.

For example, a 2 - state Markov process has its state transition diagram and the generator matrix shown in figure 6.

团 EMBED Visio. Drawing 11 团团团Q $=\left(\begin{array}{cc}-\lambda & 2 \\ g & -k\end{array}\right)$

Figure 6 Two State Systems and Generator matrix.

If we consider the probability flux in and out of state 1 , we obtain $P_{1} \lambda=R_{2} \mu$ and similarly, for state $2, R_{2} \mu=R_{1} \lambda$.

From the normalisation condition, we know that $R_{1}+R_{i}-1$.

It follows that the steady state probability distribution is
$\mathrm{P}=\left(\frac{a}{a+\lambda}, \frac{\lambda}{\beta+\lambda}\right)$. These computed steady state probabilities can now be substituted in equation 3 for evaluating $\lambda_{s i}$ and $\lambda_{s j}$.

2.1.4 Determination of the mean time to failure (first passage time)

Computing the mean time to failure (first passage time) $\mathbb{M}_{\Sigma \Sigma \Sigma 1}$, we consider first the case where there is no direct transition between states $i$ and $j$. When in state $\mathrm{S}$, the system may transfer to state i or to state $\mathrm{j}$.

Let $P_{s i}$ denote the probability of the first (transferring to state i) and

$P_{3}$ denote the probability of moving from state $\mathrm{S}$ to state $\mathrm{j}$ i.e

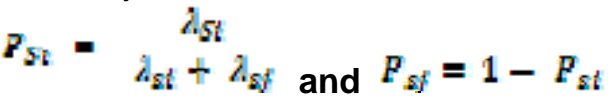

From the analysis of possible transitions [8] in Fig 5, we have

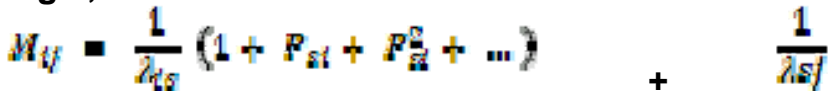$$
\left(F_{\text {si }}+F_{\text {si }} F_{\text {si }}+F_{\text {si }}^{\tau} F_{s i}^{\gamma}+\ldots\right)+\frac{1}{\lambda_{s i}}\left(F_{\text {si }}+F_{\text {si }}^{\gamma}+\ldots\right)
$$

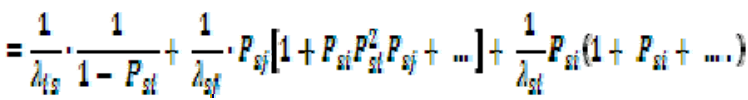$$
=
$$

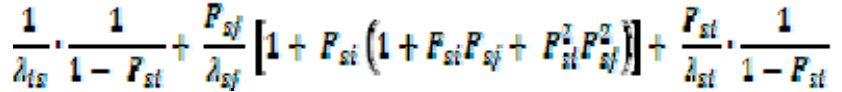

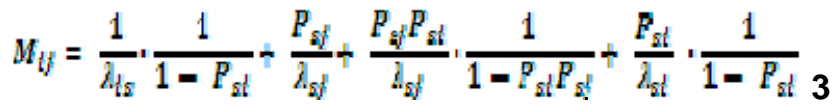


Computer program Algorithm development for the Markov model: To develop a computer program for this model, a number of questions by Utility engineers involved with maintenance planning were carefully considred. Some of the questions include:

1. In view of astronomical cost associated with maintenance, is it economical to run equipment to failure and then replace?

2. How often should the equipment be inspected to minimize the total costs of repair and maintenance?

3. Can the deterioration (ageing) process be allowed to continue for an ' $n$ ' number of years?

4. What is the average number of years for the system to transfer from the current state to a failure state?

5. What is the expected time for the insulation to be in a particular deterioration state?
6. How many years will it take for the insulation to be in a particular deterioration state for the first time given that it is in a specified state now?

7. How long, on the average, will each deterioration state last?

A structure plan of the complete algorithm for predicting the remaining life of transformer insulation as well as providing solutions to the various questions raised above is shown in figure 7 . The algorithm adopt a 'top down' approach so as to cultivate a mental discipline of getting the logic of the problem clear before attempting to develop the computer program.

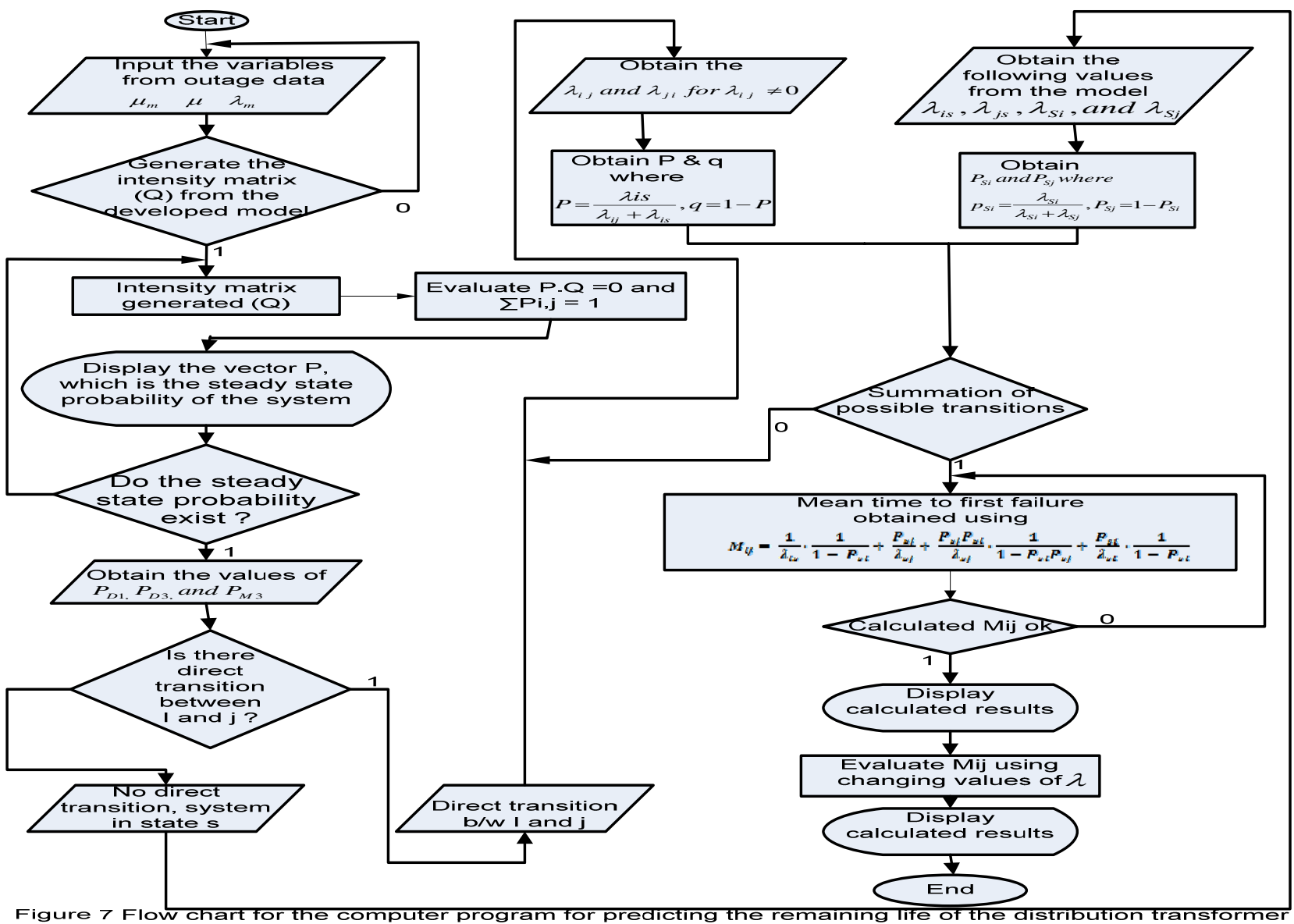


Discussion of solution obtained from computer program: The results obtained from the computer program are the esimated transformer lifespan at varying failure rate, assuming all other variable parameters such as maintenance rate $\left(c_{\mathrm{m}}\right)$ are held constant. These results are plotted as shown in figure
8. This plotted graph is fitted to $8^{\text {th }}$ degree polynomial. The resulting graph and its bar chart plots are shown in figures $9 \mathrm{a}$ and $9 \mathrm{~b}$. These verified the goodness of fit of the original plotteed result obtained from the computer program.

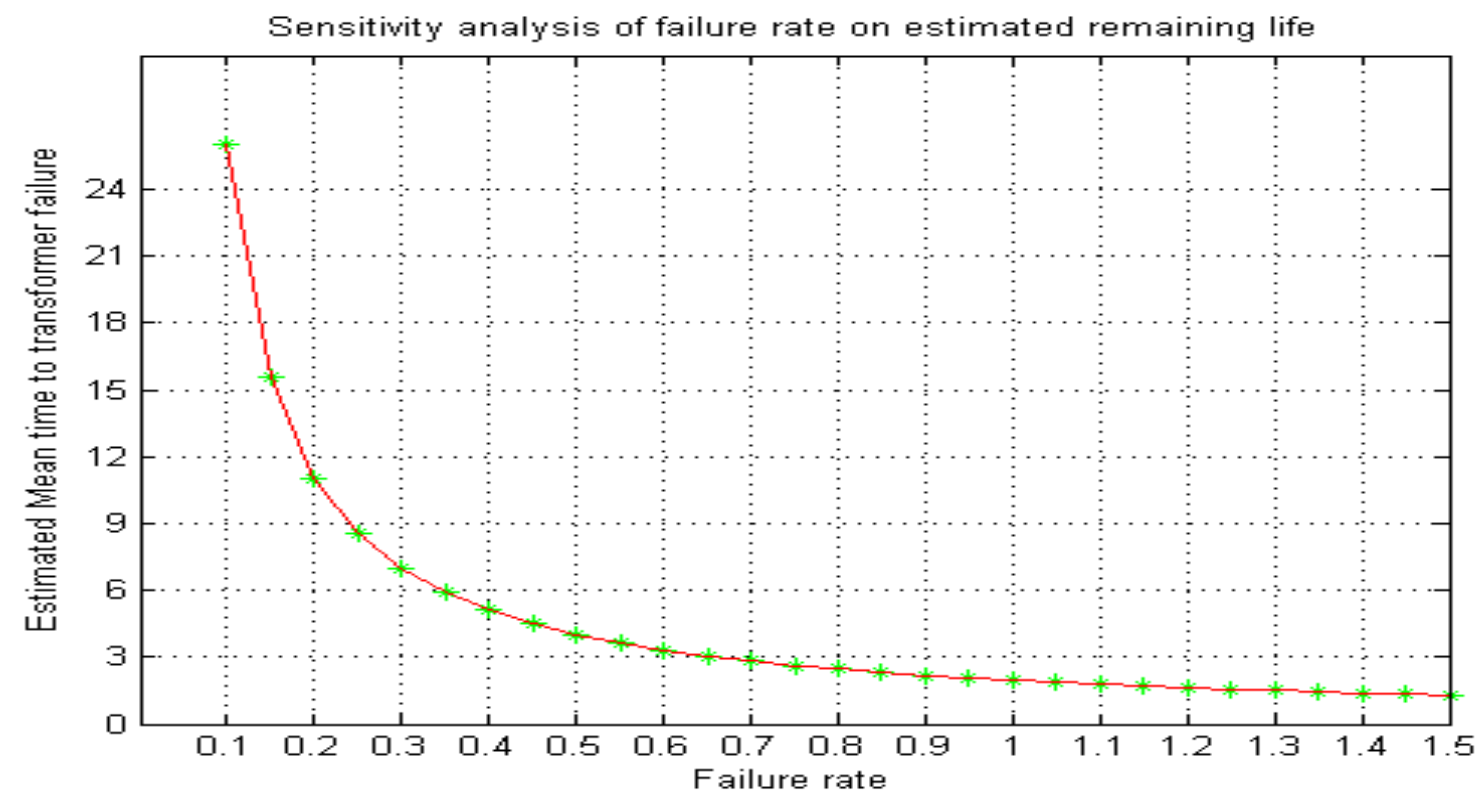

Fig 8 Estimated transformer lifespan at varying failure rate.

The next step was to hold the failure rate ( $\lambda$ ) constant and vary the maintenance rate $\left(s_{m}\right)$, the remaining life of the distributed transformer insulation was again computed from the developed computer program.

The results obtained were also plotted and the graphical representation that shows the sensitivity of this variation with the computed remaining life of the component (distributed transformer) is also shown in figure 10. This graph shows that the maintenance rate $(F m i)$ is directly proportional to the computed remaining life of the component under consideration. The linear relationship between the maintenance rate and the computed remaining life becomes more pronounced when the computed values are fitted usng Matlab tool to $3^{\text {rd }}$ degree polynomial. The goodness of fit is also displayed in the bar chart plot shown in figures $11 \mathrm{a}$ and $11 \mathrm{~b}$. The value obtained from norm (the square root of the sum of the squares of the residuals) indicated a very good fit. 


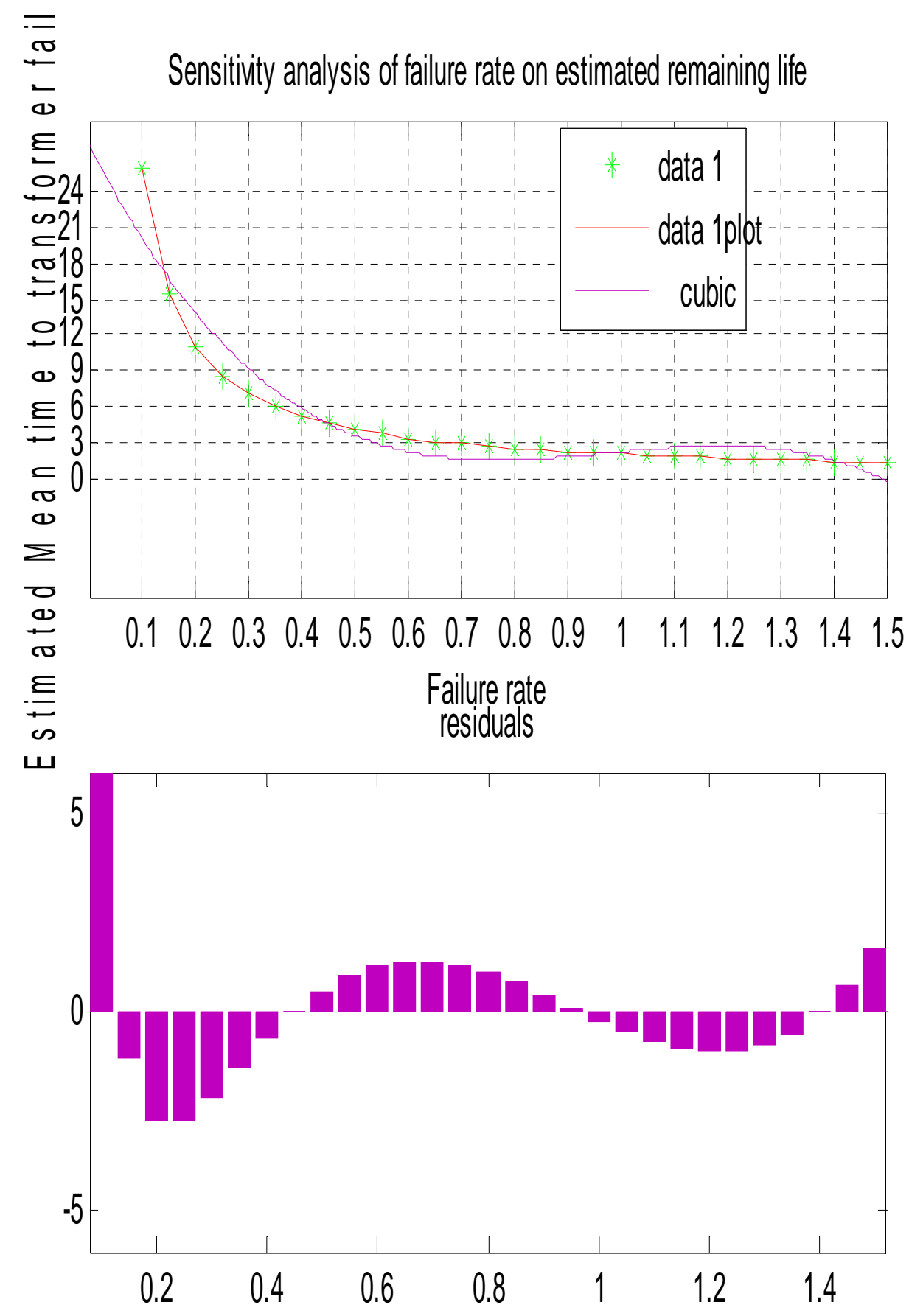

Fig 9 a-b sensitivity data fitted to $8^{\text {th }}$ degree polynomial and its corresponding Norm residuals 
Am. J. Sci. Ind. Res., 2010, 1(3): 539-548

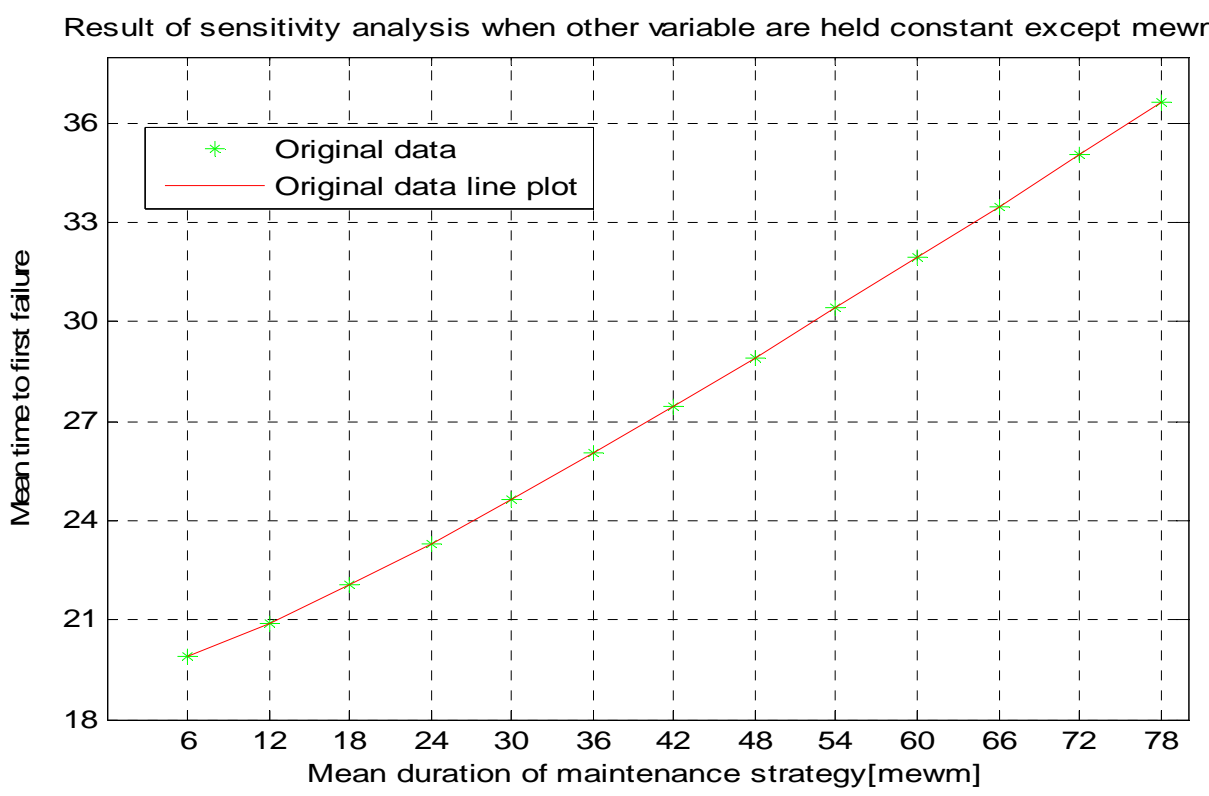

Fig 10 Plot of the result of the sensitivity analysis when other variables are held constant except $\mu_{m}$.

Result of sensitivity analysis when other variable are held constant except mewm
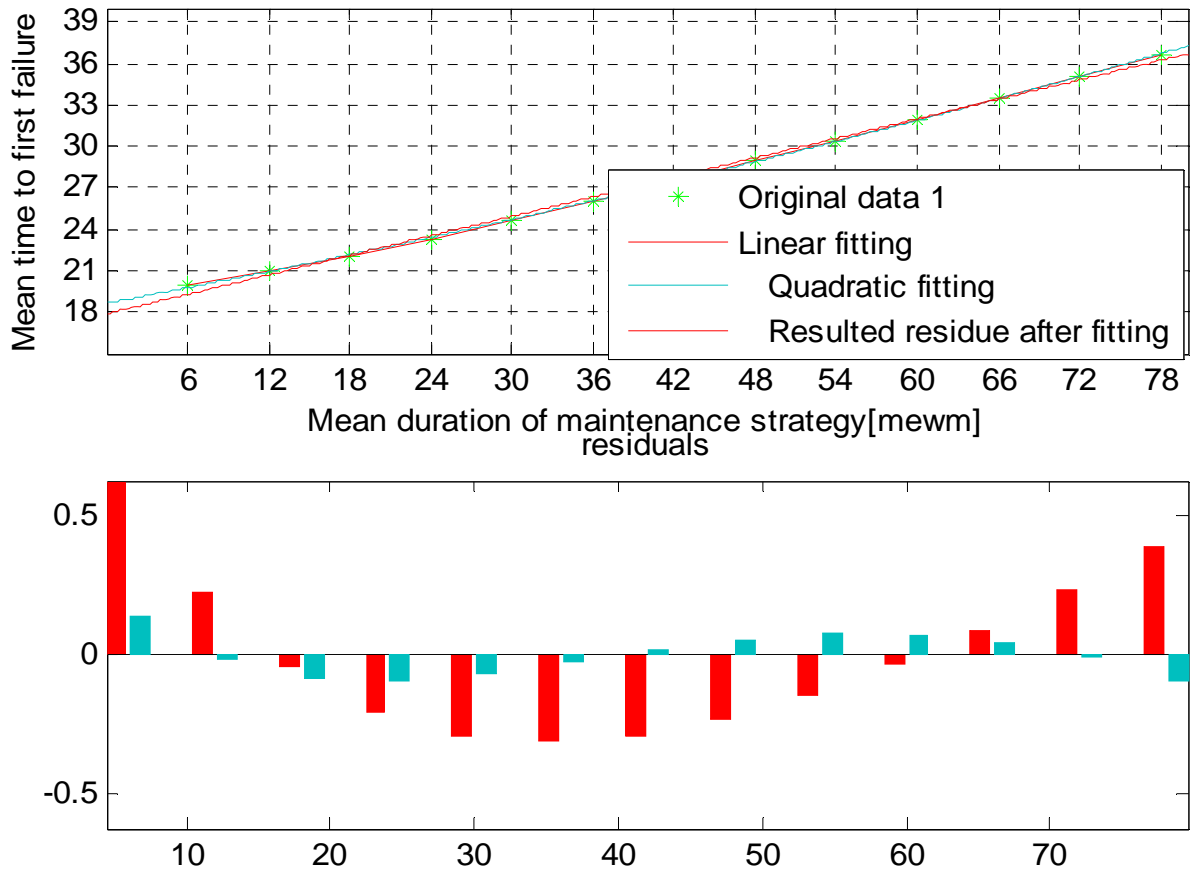

Fig 11 a-b sensitivity data fitted to $3^{\text {th }}$ degree polynomial and its corresponding Norm residuals 
Conclusions: A Markov Model for predicting the remaining life of Electrical insulation of transformer had been developed. A computer program based on new mathematical formulation of the expected transition time from any deterioration state to the failure state (expected remaining life) has been implemented. The program was applied to a distribution transformer identified as critical component in one of the Power Holding Company of Nigeria [PHCN] PLC Utility.

The main strength of this program is that it allows one to assess the state of insulation of several different groups of transformers relative to each other. It is a fact of life that prediction (forecast) stand on a firmer ground if it is relative rather than absolute.

\section{REFERENCES}

[1] Bertling, L., Allan, R., \&Eriksson, R., "A ReliabilityCentered Asset Maintenance Method for assessing the impact of maintenance in Power Distribution Systems" IEEE Transactions of power systems. Vol. 20, No 1, Feb. 2005.

[2] Brown, R.E. "Identifying Worst Performing Feeders" $8^{\text {th }}$ International conference on probabilistic methods applied to power systems, lowa state University, September 12-16, 2004
[3] Ogujor, E.A and Kuale, P.A. "Using Reliability IndicesMarkov Model in Electric Power Distribution Reliability Assessment"International Journal of Electrical and Power Engineering 1 (4) 416 - 420, Medwell Journals, 2007.

[4] Yujia, Z, Anil P, and Shie-shien, Y. "Modeling WheatherRelated Failures of Overhead Distribution Lines" IEEE Transactions on Power Systems, Vol.21,No.4, November 2006.

[5] Morcous, G. and Akhnoukh, A. "Stochastic Modeling of Infrastructure Deterioration: An Application to Concrete Bridge Decks" Joint International Conference on computing and Decision Making in Civil and Building Engineering, June 14-16, 2006, Montreal, Canada.

[6] Dongyan, C and Kishor, S.T. "Optimization for conditionbased maintenance with semi-Markov decision process" ELSEVIER, Reliability Engineering and system safety, 90 (2005) 25-29.

[7] Awosope, C.O.A and Akinbulire, T.O. "A Computer program for Generating Power-System Load-Point Minimal paths"IEEE Transactions on Reliability, Vol.40, No.3, August,1991.

[8] Anders, S.M, Endrenyi, F, Ford, G.L and Stone, G.C. "A Probabilistic Model for evaluating the remaining life of Electrical Insulation in Rotating Machines" IEEE Transactions on Energy Conversion, Vol. 5, No. 4, December 1990. 